\title{
TEM Investigations of Pt-NPs Loaded Fibrous Nano-Catalyst Support KCC-1
}

\author{
Dalaver H. Anjum ${ }^{1}$ and Pradip Sarawade ${ }^{2}$ \\ 1. Imaging and Characterization Lab, King Abdullah University of Science \& Technology (KAUST), \\ Thuwal, Kingdom of Saudi Arabia (KSA) \\ 2. KAUST Catalysis Center (KCC), KAUST, Thuwal, KSA
}

Nanocatalysts include solid-state heterogeneous catalysts whose dimensions are in the nanometer length scale [1]. This type of catalysts can decrease the energy usage in the chemical processes that ultimately leads to a greener chemical industry. There are many different types of supports that are used to carry these catalysts but generally the ones with higher surface area are preferred because it results in an improved catalytic activity. Silica with mesoporous structure $\left(\mathrm{mSiO}_{2}\right)$ is an example of such high surface area containing support. However it has been recently shown that the fibrous silica supports possess even higher surface area than their mesoporous counterparts and hence perform better in the catalytic processes $[2,3]$. In this study, we carried out a TEM analysis of such a fibrous silica support called KCC-1 which has been loaded with platinum nanoparticles (Pt-NPs). The objective of the study was to investigate the quality of Pt-NPs loading on the surface of KCC-1 as well as to determine the average size and the structure of Pt-NPs. We accomplish this by utilizing the bright-field TEM (BFTEM), electron tomography, and aberration-corrected scanning TEM (AC-STEM) techniques.

Figure 1A contains a BF-TEM micrograph of about $250 \mathrm{~nm}$ size $\mathrm{KCC}-1$ particle which is loaded with Pt-NPs. Micrograph is acquired with FEI Company's Titan $\mathrm{G}^{2} 80-300 \mathrm{CT}^{\mathrm{microscope}}$ by operating it at a beam energy of $300 \mathrm{keV}$. A uniform loading of Pt-NPs with an average size of about $1.2 \mathrm{~nm}$ on to the surface of KCC-1 particle can be seen in the micrograph. Nevertheless the image contrast (massthickness contrast in this case) was not able to show a clear image of smaller size ( $\sim 0.6 \mathrm{~nm}$ or less) PtNPs as they might be mistakenly considered as amorphous contrast emanating from $\mathrm{mSiO} 2$. The quality of Pt-NPs loading on the same KCC-1 particle was further investigated with BF-tomography analysis which was carried out in the same microscope. During the tomography data-acquisition, the specimen was tilted in the range of $+/-70^{\circ}$ with an increment of $1^{\circ}$. The acquired data (or tomoseries) was then aligned and reconstructed to generate the tomogram of the KCC-1 particle. Figure 1B-D contains the volume slices that are extracted from the bottom, middle, and top regions of the KCC-1 particle. These volume slices clearly show the presence of Pt-NPs in the entire structure of KCC-1 particle. This type of ubiquitous decoration of KCC-1 particles with Pt-NPs implies a higher catalytic activity than conventional catalyst-supports. The crystal structure of Pt-NPs was investigated by performing the ACSTEM analysis of samples. Figure 2A contains a low magnification HAADF-STEM micrograph. It was acquired by using FEI Company's Cs-probe corrected Titan $\mathrm{G}^{2}$ 60-300 ST microscope at $300 \mathrm{keV}$ as well. Image contrast (or z-contrast in this case) of Pt-NPs sitting on KCC-1 is more enhanced in comparison to the contrast in Figure 1A. As a result of this, the identification of the Pt-NPs sitting on the surface of KCC-1 became more intuitive and less cumbersome. In fact, a higher magnification HAADFSTEM micrograph (Figure 2B) not only provided a much clearer distinction of less than one nm size PtNPs but it also showed the presence of single Pt-atoms on the surface of KCC-1. It further showed that the Pt-NPs of size about $1 \mathrm{~nm}$ or larger crystalize with having FCC crystal structure. Overall, it can be concluded from the TEM study that a uniform surface loading of small size Pt-NPs (as well as single Pt-atoms) on KCC-1 particles is accomplished and hence higher catalytic activity can be achieved by using this nano-catalvst material. 


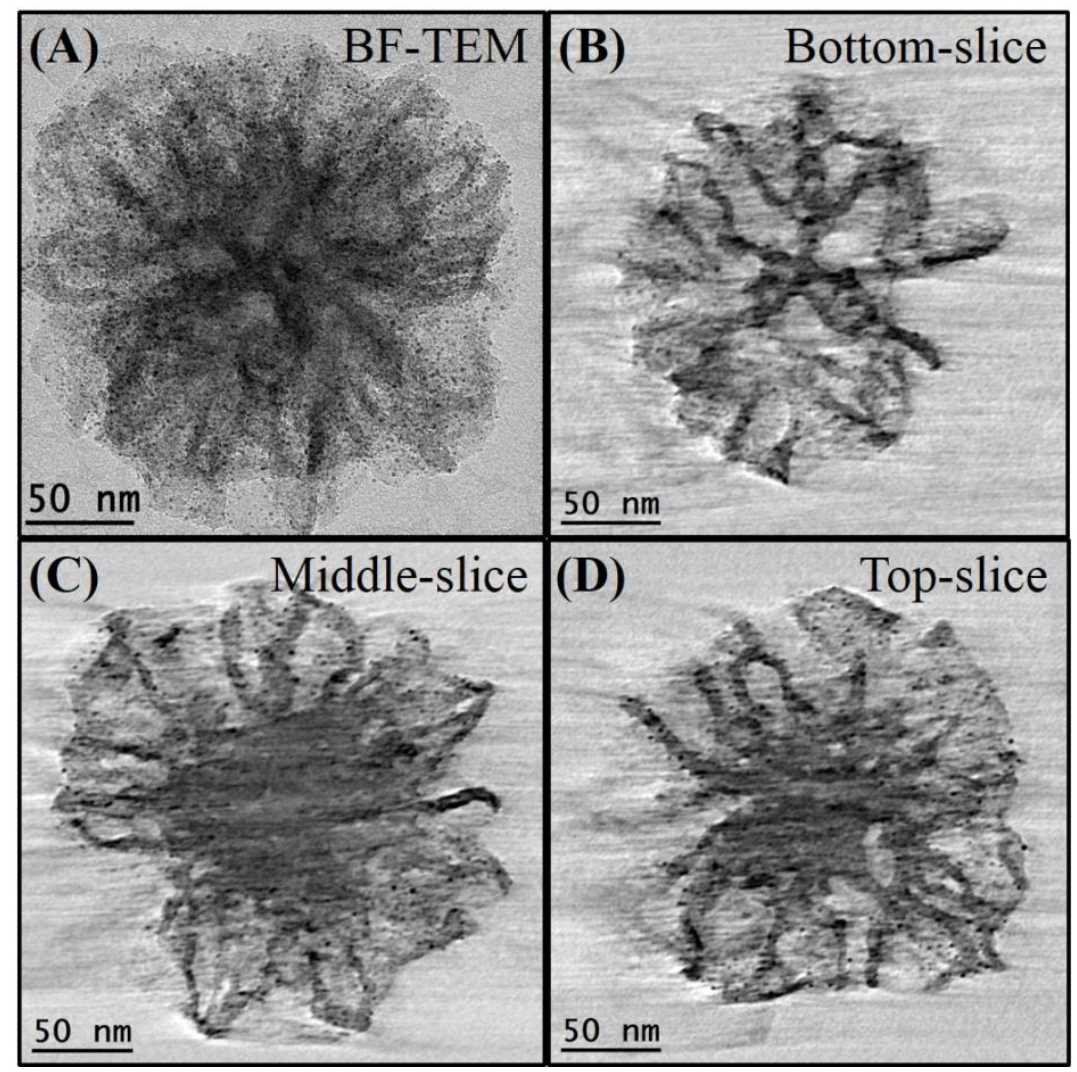

Figure 1: BF-TEM and tomography analysis of Pt-NPs loaded KCC-1 particle

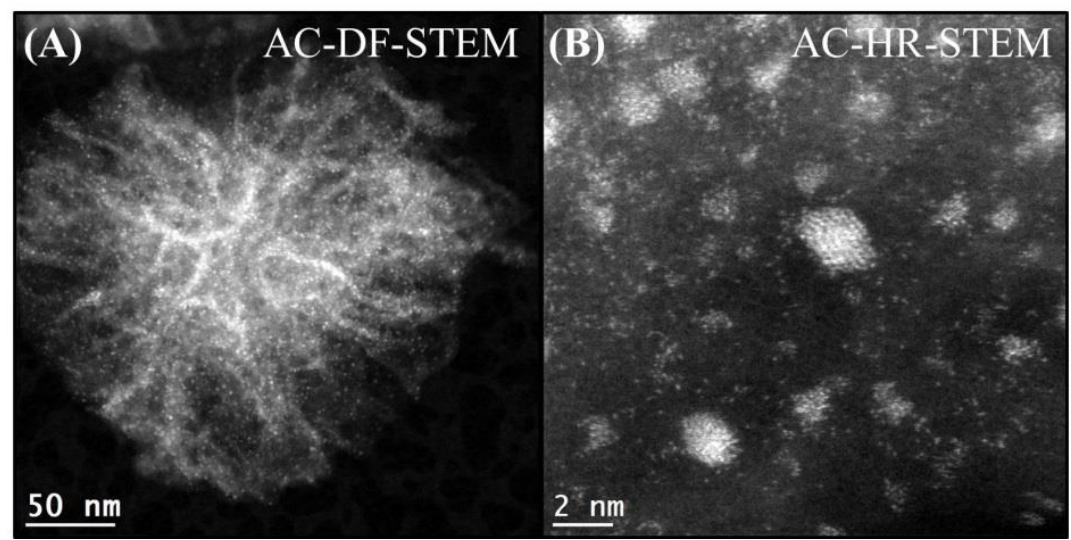

Figure 2: AC-STEM analysis of Pt-NPs loaded KCC-1 particle

References:

[1] G. A. Somorjai et al., PNAS, 103 (28), 10577-10583 (2006).

[2] V. Polshettiwar et al., Angew. Chem. Int. Ed. 49, 9652-9556 (2010).

[3] A. Fihri et al., ACS Catalysis, 2, 1425-1431 (2012). 